\title{
CTEQ-TEA parton distribution functions with intrinsic charm
}

\author{
Marco Guzzi ${ }^{1, *}$, Tie-Jiun $\mathrm{Hou}^{2,6}$, Sayipjamal Dulat ${ }^{3}$, Jun $\mathrm{Gao}^{4}$, Joey Huston ${ }^{5}$, Pavel \\ Nadolsky ${ }^{6}$, Carl Schmidt ${ }^{5}$, Jan Winter ${ }^{5}$, Keping Xie ${ }^{6}$, and C.-P. Yuan ${ }^{5}$ \\ ${ }^{1}$ Department of Physics, Kennesaw State University, 370 Paulding Ave., 30144 Kennesaw, GA, U.S.A. \\ ${ }^{2}$ National Tsing Hua University, Hsinchu City, Taiwan. \\ ${ }^{3}$ School of Physics Science and Technology \& Center for Theoretical Physics, Xinjiang University, \\ Urumqi, Xinjiang 830046 China. \\ ${ }^{4}$ INPAC, Shanghai Key Laboratory for Particle Physics and Cosmology \\ \& School of Physics and Astronomy, Shanghai Jiao Tong University, Shanghai 200240, China. \\ ${ }^{5}$ Department of Physics and Astronomy, Michigan State University, East Lansing, MI 48824 U.S.A. \\ ${ }^{6}$ Department of Physics, Southern Methodist University, Dallas, TX 75275-0181, U.S.A.
}

\begin{abstract}
We present a study in which the possibility of a (sizable) nonperturbative contribution to the charm parton distribution function (PDF) in a nucleon is investigated together with theoretical issues arising in its interpretation. The separation of the universal component of the nonperturbative charm from the rest of the radiative contributions is also discussed. We illustrate the potential impact of a nonperturbative charm PDF on LHC scattering processes. An estimate of nonperturbative charm magnitude in the CT14 and CT14HERA2 global QCD analyses at the next-to-next-to leading order (NNLO) in the QCD coupling strength is given by including the latest experimental data from HERA and the Large Hadron Collider. We show a comparison between different models of intrinsic charm and illustrate prospects for standard candle observables at the LHC.
\end{abstract}

\section{Introduction}

The principle of the global analysis is to use QCD theory to analyze a broad range of experimental data, including precision data from HERA, the Tevatron, and the Large Hadron Collider (LHC). In particular, theoretical predictions for short-distance scattering processes allow the measurement, within some approximations, of universal parton distribution functions (PDFs) for the proton. As new experimental measurements from the LHC grow increasingly precise, novel challenges arise in extracting accurate predictions for the parton content of the proton in global QCD analysis of PDFs needed for advanced tests of the Standard Model and possible physics beyond the Standard Model. A recently published CTEQ-TEA (CT) global analysis of experimental data [1] produced the CT14NNLO PDFs, referred to as the CT14 PDFs. The analysis is based on the next-to-next-to-leading order (NNLO) approximation for perturbative QCD.

\footnotetext{
*e-mail: mguzzi@kennesaw.edu
} 


\subsection{The perturbative charm PDF}

In global PDF analyses, all QCD parameters, such as $\alpha_{s}$ and the quark masses, are correlated with the PDFs. The determination of the PDFs depends not only on the data sample included in the fits, but also on the specific theory assumptions and underlying physics models. As one such choice made in the standard CT PDF sets, the charm quark and antiquark PDFs are taken to be zero below a low energy scale $Q_{c}=Q_{0}$ of order of the charm mass. In the CT14 analysis, the charm quark and antiquark PDFs were turned on at the scale $Q_{c}=Q_{0}=m_{c}=$ $1.3 \mathrm{GeV}$, with an initial $O\left(\alpha_{s}^{2}\right)$ distribution consistent with NNLO matching [2,3] to the threeflavor result. At higher $Q$, most of the charm PDF is generated from the DGLAP evolution that proceeds through perturbative splittings of gluons and light-flavor quarks. Hence, the charm PDF from a standard global analysis is called "perturbative" and it is obtained by perturbatively evolving the PDFs from the initial scale $Q_{c}$ to the experimental data scale $Q$.

\subsection{A nonperturbative component for the charm PDF}

In addition to the perturbative charm production mechanism, it is believed that "intrinsic charm quarks" may emerge from the nonperturbative structure of the hadronic bound state. The plausibility of the intrinsic charm (IC) component, its dynamical origin, and its actual magnitude have been a subject of a long-standing debate. Indeed, QCD theory rigorously predicts existence of power-suppressed (higher-twist) channels for charm quark production that are independent of the leading-power (twist-2, or perturbative) production of charm quarks. The IC quarks have been associated with the excited $|u u d c \bar{c}\rangle$ Fock state of the proton wave function [4-9] and predicted by meson-baryon models [10-13]. The range of validity of the PDF models with nonperturbative charm has been appraised in a recent $\mathrm{CT}$ analysis published in Ref. [14] and in other recent studies [15-19].

\subsection{Fitted charm and nonperturbative charm parametrizations}

Starting from the factorization theorem for DIS cross sections with massive fermions, that is a fundamental QCD result, one can draw a consequential distinction between the "fitted" charm PDF parametrization and the nonperturbative charm PDF. The fitted charm PDF accounts for the nonperturbative charm plus other (possibly not universal) higher $O\left(\alpha_{s}\right)$ higher power suppressed terms. Since the perturbative charm PDF component cancels near the threshold up to a higher order, the fitted charm component may approximate for a missing higher-order term or a power-suppressed nonperturbative component. The genuine nonperturbative charm PDF instead, is defined by the means of power counting of radiative contributions to DIS. Assuming that this additional nonperturbative charm component can be factorized like the perturbative charm component, one is able to examine how it differs from the perturbative charm, and how it depends on theoretical inputs in a global QCD analysis of PDFs. In principle, the intrinsic charm content would be suppressed by powers of $\left(\Lambda_{\mathrm{QCD}}^{2} / m_{c}^{2}\right)$, but, since this ratio is not very small, it may be relevant in some processes such as precise DIS. For a more detailed description of QCD factorization with power suppressed charm contributions, we refer the reader to the recent CTEQ-TEA analysis of Ref. [14].

\subsection{Valence-like and sea-like models for the charm PDF parametrization}

Various model estimates suggest a power-suppressed charm cross section of a modest size: of order of a fraction of the $\alpha_{s}^{2}$ component in DIS charm production, carrying less than about a percent of the proton's momentum. We examine a more extensive list of nonperturbative 
models, fit the most complete set of DIS data from HERA as well as the data from the LHC and (optionally) the EMC experiment [20], and utilize a PDF parametrization that results in a more physical behavior. Given that several mechanisms may give rise to the fitted charm, we will parametrize it by two generic shapes, a "valence-like" and a "sea-like" shape. The two shapes arise in a variety of dynamical models.

A valence-like shape has a local maximum at $x$ above 0.1 and satisfies $f_{q / p}\left(x, Q_{c}\right) \sim$ $x^{-a_{1}}$ with $a_{1} \lesssim 1 / 2$ for $x \rightarrow 0$ and $f_{q / p}\left(x, Q_{c}\right) \sim(1-x)^{a_{2}}$ with $a_{2} \gtrsim 3$ for $x \rightarrow 1$. The distributions for valence $u$ and $d$ quarks fall into this broad category, as well as the "intrinsic" sea-quark distributions that can be naturally generated in several ways [6], e.g., for all flavors, nonperturbatively from a $|u u d Q \bar{Q}\rangle$ Fock state in light-cone [4, 5, 7-9] and meson-baryon models [10-13]; for $\bar{u}$ and $\bar{d}$, from connected diagrams in lattice QCD [21]. ${ }^{1}$ The approximate Brodsky-Hoyer-Peterson-Sakai (BHPS) model $[4,5]$ parametrizes the charm PDF at $Q_{0}$ by a valence-like nonperturbative function

$$
\widehat{c}(x)=\frac{1}{2} A x^{2}\left[\frac{1}{3}(1-x)\left(1+10 x+x^{2}\right)-2 x(1+x) \ln (1 / x)\right] .
$$

This function is obtained from a light-cone momentum distribution by taking the charm mass to be much heavier than the masses of the proton and light quarks: $m_{c} \gg M_{p}, m_{u}, m_{d} . A$ is the normalization factor that is to be determined from the fit. The BHPS1 and BHPS2 global fits are obtained with this parametrization choice and are illustrated in Sec. 2 below.

Instead of approximating the probability integral as in the original BHPS model, the $\widehat{c}(x)$ can also be obtained by solving the BHPS model for the $|u u d c \bar{c}\rangle$ Fock state numerically and keeping the exact dependence on $M_{p}, m_{u}$, and $m_{d}$. In this BHPS model, the intrinsic quark distributions are determined by starting from a $|u u d q \bar{q}\rangle$ proton Fock state, where the probability differential for a quark $i$ to carry a momentum fraction $x_{i}$ is given by

$$
d \mathcal{P}\left(x_{1}, \ldots, x_{5}\right)=A d x_{1} \ldots d x_{5} \delta\left(1-\sum_{i=1}^{5} x_{i}\right) \frac{1}{\left[M_{p}^{2}-\sum_{i=1}^{5} \frac{m_{i}^{2}}{x_{i}^{2}}\right]^{2}} .
$$

This generalized BHPS model, used in the context of the CT14HERA fit with IC, is named BHPS3. We do not, however, include the intrinsic contribution to the $s$ quark PDF, because it is overwhelmed by the very large strange PDF uncertainty. The presence of an intrinsic component for the strange quark does not affect our conclusions about the nonperturbative charm.

A sea-like component is usually monotonic in $x$ and satisfies $f_{q / p}\left(x, Q_{c}\right) \sim x^{-a_{1}}$ for $x \rightarrow 0$ and $f_{q / p}\left(x, Q_{c}\right) \sim(1-x)^{a_{2}}$ for $x \rightarrow 1$, with $a_{1}$ slightly above 1 , and $a_{2} \gtrsim 5$. This behavior is typical for the leading-power, or "extrinsic" production. For example, an (anti)quark PDF with this behavior originates from $g \rightarrow q \bar{q}$ splittings in perturbative QCD, or from disconnected diagrams in lattice QCD (see Ref. [21] for details). Even a missing next-to-next-tonext-to-leading order (N3LO) leading-power correction may produce a sea-like contribution at $x \ll 0.1$, where the valence-like components are suppressed. In the SEA model, the charm PDF is parametrized by a "sea-like" nonperturbative function that is proportional to the light quark distributions:

$$
\widehat{c}(x)=A\left(\bar{d}\left(x, Q_{0}\right)+\bar{u}\left(x, Q_{0}\right)\right) .
$$

This model is assumed with the SEA1 and SEA2 PDF sets which are illustrated in Sec. 2 below. Finally, the normalization coefficient $A$ in the models described above can be derived

\footnotetext{
${ }^{1}$ In contrast to the light flavors, in lattice QCD a charm PDF arises exclusively from disconnected diagrams [22]. This suggests that $c$ and $\bar{c}$ contributions in DIS are connected to the hadron target by gluon insertions.
} 

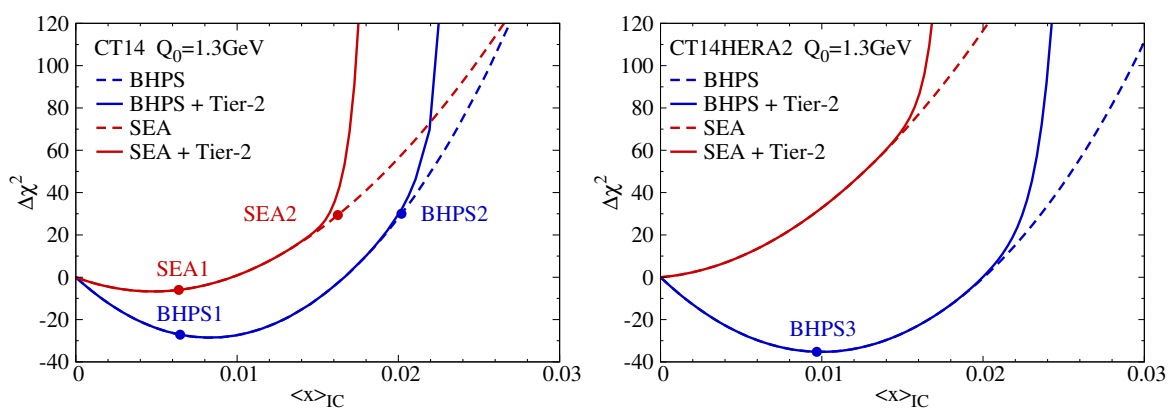

Figure 1. The change $\Delta \chi^{2}$ in the goodness of fit to the CT14 (left) and CT14HERA2 (right) data sets as a function of the charm momentum fraction $\langle x\rangle_{\text {IC }}$ for the BHPS (blue) and SEA (red) models. Solid (dashed) lines represent the total $\chi^{2}$ and the partial $\chi_{\text {global }}^{2}$, as defined in Sec. 2.

from the charm momentum fraction (first moment) at scale $Q$ :

$$
\langle x\rangle_{\mathrm{IC}}=\int_{0}^{1} x\left[c\left(x, Q_{0}\right)+\bar{c}\left(x, Q_{0}\right)\right] d x .
$$

By its definition, $\langle x\rangle_{\text {IC }}$ is evaluated at the initial scale $Q_{0}$. It is to be distinguished from the full charm momentum fraction $\langle x\rangle_{c+\bar{c}}(Q)$ at $Q>Q_{c}$, which rapidly increases with $Q$ because of the admixture of the twist-2 charm component.

\section{Results of the global analysis}

In this section we illustrate the main findings of our global analysis in which we assume that the additional nonperturbative charm component can be factorized in a similar fashion to the perturbative one. To gauge the preference of the global QCD data to a specific $\langle x\rangle_{\mathrm{IC}}$, we examine the goodness-of-fit function $\chi^{2} \equiv \chi_{\text {global }}^{2}+P$, constructed in the CT14 method from the global $\chi_{\text {global }}^{2}$ and a "tier-2" statistical penalty $P[1]$. It is convenient to compare each fit with an $\langle x\rangle_{\mathrm{IC}} \neq 0$ to the "null-hypothesis" fit obtained assuming $\langle x\rangle_{\mathrm{IC}}=0$. Thus, we start by computing $\Delta \chi^{2} \equiv \chi^{2}-\chi_{0}^{2}$, where $\chi^{2}$ and $\chi_{0}^{2}$ are given for $\langle x\rangle_{\mathrm{IC}} \neq 0$ and $\langle x\rangle_{\mathrm{IC}}=0$, respectively, at 50 values of $\langle x\rangle_{\text {IC }}$ and default $Q_{0}=m_{c}^{\text {pole }}=1.3 \mathrm{GeV}$. We plot the resulting $\Delta \chi^{2}$ behavior in Fig. 1. The CT14 (CT14HERA2) data sets are compared against the approximate (exact) solution of the BHPS model, respectively. The SEA charm parametrizations are constructed as in Eq. (3) in terms of the respective CT14 or CT14HERA2 light-antiquark parametrizations. We see from Fig. 1 that large amounts of intrinsic charm are disfavored for all models under scrutiny. A mild reduction in $\chi^{2}$, however, is observed for the BHPS fits, roughly at $\langle x\rangle_{\text {IC }}=1 \%$, both in the CT14 and CT14HERA2 frameworks. The significance of this reduction and the upper limit on $\langle x\rangle_{\text {IC }}$ depends on the assumed criterion. In CTEQ practice, a set of PDFs with $\Delta \chi^{2}$ smaller (larger) than 100 units is deemed to be accepted (disfavored) at about $90 \%$ C.L. Thus, a reduction of $\chi^{2}$ by less than forty units for the BHPS curves has significance roughly of order one standard deviation. The new upper limits on $\langle x\rangle_{\mathrm{IC}}$ in the CT14 and CT14HERA2 analyses at the 90\% C.L.: $\langle x\rangle_{\text {IC }} \lesssim 0.021$ for CT14 BHPS; $\langle x\rangle_{\text {IC }} \lesssim$ 0.024 for CT14HERA2 BHPS; $\langle x\rangle_{\mathrm{IC}} \lesssim 0.016$ for CT14 and CT14HERA2 SEA. 

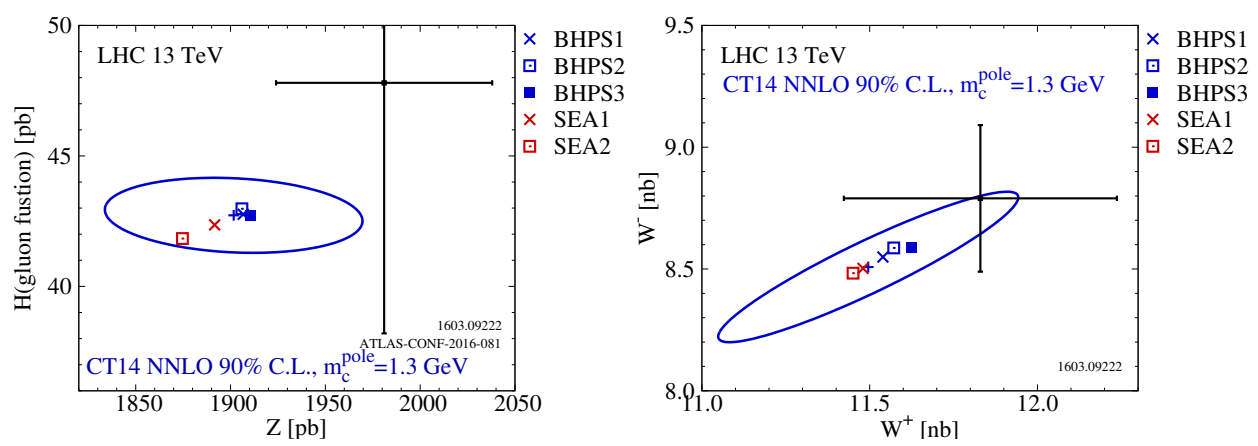

Figure 2. CT14 NNLO $H$ (gluon-gluon fusion), $Z, W+$, and $W$ - production cross sections with an IC PDF component at the LHC $\sqrt{s}=13 \mathrm{TeV}$, with pole mass $m_{c}^{\text {pole }}=1.3 \mathrm{GeV}$. The $90 \%$ C.L. uncertainty regions for CT14 at NNLO and experimental points from ATLAS [23, 24] are also shown.

\subsection{Impact of IC on the electroweak $Z$ and $H$ boson production cross sections at the LHC}

Figure 2 illustrates predictions of the total cross sections for inclusive production of electroweak bosons $W^{ \pm}, Z^{0}$, and $H$ (via gluon-gluon fusion) for the BHPS and SEA models at the LHC $\sqrt{s}=13 \mathrm{TeV}$ with charm quark mass $m_{c}=1.3 \mathrm{GeV}$. To provide a visual measure of the CT14NNLO uncertainty, each figure shows an error ellipse corresponding to CT14 NNLO at the $90 \%$ C.L. and experimental data points relative to measurements from the ATLAS collaboration [23, 24]. The $W$ and $Z$ inclusive cross sections (multiplied by branching ratios for the decay into one charged lepton flavor), are calculated by using the VRAP v0.9 program $[25,26]$ at NNLO in QCD, with the renormalization and factorization $\left(\mu_{R}\right.$ and $\left.\mu_{F}\right)$ scales set equal to the invariant mass of the vector boson. The Higgs boson cross sections via gluon-gluon fusion are calculated at NNLO in QCD by using the IHIxs v1.3 program [27], in the heavy-quark effective theory (HQET) with finite top quark mass correction, and with the QCD scales set equal to the invariant mass of the Higgs boson. The central value predictions for the BHPS and SEA models are all within the CT14 NNLO uncertainties, with BHPS very close to the CT14 nominal fit. The impact of IC on these key LHC observables is mild.

\section{Conclusions}

We explored the possibility of sizeable nonperturbative contribution to charm PDF assuming that factorization for such contributions exists. We have determined the magnitude of the IC component of the proton that is consistent with the CT14 global QCD analysis of hard scattering data: $\langle x\rangle<2 \%$ for BHPS IC and $\langle x\rangle<1.6 \%$ for SEA IC at $90 \%$ C.L.. As of today, the experimental confirmation of the IC component in the proton is still missing, and data from far more sensitive measurements are required.

Acknowledgments. This work was supported in part by the U.S. Department of Energy under Grant No. DESC0010129; by the U.S. National Science Foundation under Grant No. PHY-1417326; by the National Natural Science Foundation of China under the Grant No. 11465018; and by the Lancaster-Manchester-Sheffield Consortium for Fundamental Physics under STFC Grant No. ST/L000520/1. The work of J.G. is sponsored by Shanghai Pujiang Program. 


\section{References}

[1] S. Dulat et al., Phys. Rev. D93 (2016) 033006, 1506.07443.

[2] M. Buza et al., Nucl. Phys. B472 (1996) 611, hep-ph/9601302.

[3] M. Buza et al., Eur. Phys. J. C1 (1998) 301, hep-ph/9612398.

[4] S.J. Brodsky et al., Phys. Lett. B93 (1980) 451.

[5] S.J. Brodsky, C. Peterson and N. Sakai, Phys. Rev. D23 (1981) 2745.

[6] J. Pumplin, Phys. Rev. D73 (2006) 114015, hep-ph/0508184.

[7] W.C. Chang and J.C. Peng, Phys. Rev. Lett. 106 (2011) 252002, 1102.5631.

[8] J. Bluemlein, Phys. Lett. B753 (2016) 619, 1511.00229.

[9] S.J. Brodsky et al., Adv. High Energy Phys. 2015 231547, (2015) 1504.06287.

[10] F.S. Navarra et al., Phys. Rev. D54 (1996) 842, hep-ph/9504388.

[11] S. Paiva et al., Mod. Phys. Lett. A13 (1998) 2715, hep-ph/9610310.

[12] F.M. Steffens, W. Melnitchouk and A.W. Thomas, Eur. Phys. J. C11 (1999) 673, hep$\mathrm{ph} / 9903441$.

[13] T.J. Hobbs, J.T. Londergan and W. Melnitchouk, Phys. Rev. D89 (2014) 074008, 1311.1578.

[14] T.J. Hou, S. Dulat, J. Gao, M. Guzzi, J. Huston, P. Nadolsky, C. Schmidt, J. Winter, K. Xie, and C. -P. Yuan JHEP 1802 (2018) 059, 1707.00657.

[15] P. Jimenez-Delgado et al., Phys. Rev. Lett. 114 (2015) 082002, 1408.1708.

[16] P. Jimenez-Delgado et al., Phys. Rev. Lett. 116 (2016) 019102, 1504.06304.

[17] F. Lyonnet et al., JHEP 07 (2015) 141, 1504.05156.

[18] NNPDF, R.D. Ball et al., Eur. Phys. J. C76 (2016) 647, 1605.06515.

[19] NNPDF, R.D. Ball et al., (2017), Eur.Phys.J. C77 (2017) no.10, 6631706.00428.

[20] European Muon, J.J. Aubert et al., Nucl. Phys. B213 (1983) 31.

[21] K.F. Liu et al., Phys. Rev. Lett. 109 (2012) 252002, 1206.4339.

[22] K.F. Liu, private communication.

[23] ATLAS, G. Aad et al., Phys. Lett.B759 (2016) 601, 1603.09222.

[24] ATLAS Collaboration, ATLAS-CONF-2016-081.

[25] C. Anastasiou et al., Phys. Rev. D69 (2004) 094008, hep-ph/0312266.

[26] C. Anastasiou et al., Phys. Rev. Lett. 91 (2003) 182002, hep-ph/0306192.

[27] C. Anastasiou et al., JHEP 12 (2011) 058, 1107.0683. 